\title{
Curvature Dependent Skeletonization
}

\author{
Andrea Torsello and Edwin R. Hancock \\ Department of Computer Science, \\ University of York, \\ York, YO10 5DD, UK
}

\begin{abstract}
The Hamilton-Jacobi approach has proved to be a powerful and elegant method for extracting the skeleton of a shape. The approach is based on the fact that the dynamics of the inward evolving boundary is conservative everywhere except at skeletal points. Nonetheless this method appears to overlook the fact that the linear density of the evolving boundary front is not constant where the front is curved. In this paper we present an analysis which takes into account variations of density due to boundary curvature. This yields a skeletonization algorithm that is both better localized and less susceptible to boundary noise than the Hamilton-Jacobi method.
\end{abstract}

\section{Introduction}

The skeletal abstraction of $2 \mathrm{D}$ and $3 \mathrm{D}$ objects has proved to be an alluring yet highly elusive goal for over 30 years in shape analysis [2]. The morphological skeleton of a shape is defined as the set of singularities in the inward evolution of the boundary with constant velocity. The dynamics of the boundary is described by the eikonal equation: a partial differential equation that governs the motion of a wave-front through a medium. In the case of a uniform medium the equation is $\partial_{t} \boldsymbol{C}(t)=\alpha \boldsymbol{N}(t)$, where $\boldsymbol{C}(t):[0, s] \rightarrow \mathbb{R}^{2}$ is the equation of the front at time $t$ and $\boldsymbol{N}(t):[0, s] \rightarrow \mathbb{R}^{2}$ is the equation of the normal to the wave front in the direction of motion and $\alpha$ is the propagation speed. As the wave front evolves, opposing segments of the wave-front collide, generating a singularity.

Broadly speaking, the there are three approaches to skeleton extraction, namely a) marching front methods which simulate the grassfire transform which involve either thinning [1] or curve evolution [9] b) Voronoi triangulation methods $[6,4]$ and c) methods based on the differential geometry of the object boundary. In this paper, we are interested in this latter class of methods. Here a recently developed and particularly powerful method is that based on the differential equation which arises when the object boundary evolves under the HamiltonJacobi equations of classical mechanics [5]. Where resulting eikonal equation for the motion flow field is non-singular, the system is Hamiltonian, and, thus, conservative. Wherever the system ceases to be conservative there is a singularity in the boundary flow field, and when the boundary reaches the singularity a so-called shock forms. In the Hamilton-Jacobi framework skeletal points are detected by searching for points where the system ceases to be Hamiltonian, that 
is points where the divergence of the flow is not 0 [7]. These methods turn out to be algorithmically very simple and numerically stable.

A major problem of the Hamilton-Jacobi method is that in its original implementation, is that it overlooks the fact that the density of the evolving boundary front is not constant and, infact, depends on the curvature of the front. As a result of the variations in density, the flux is not conserved and hence the whole premise of the skeletonization method collapses. In this paper, we address this problem by extending the Hamilton-Jacobi analysis to the case where the front density varies due to boundary curvature. The main practical advantage of this analysis is that it leads to the recovery or more stable skeletons.

\section{Hamilton-Jacobi Skeleton}

We commence by defining a distance-map that assigns to each point on the interior of an object the closest distance $D$ from the point to the boundary (i.e. the distance to the closest point on the object boundary). The gradient of this distance-map defines a field $\boldsymbol{F}$ whose domain is the interior of the shape. The field is defined to be $\boldsymbol{F}=\nabla D$, where $\nabla=\left(\frac{\partial}{\partial x}, \frac{\partial}{\partial y}\right)^{T}$ is the gradient operator. The trajectory followed by each boundary point under the eikonal equation can be described by the ordinary differential equation $\dot{\boldsymbol{x}}=\boldsymbol{F}(\boldsymbol{x})$, where $\boldsymbol{x}$ is the coordinate vector of the point. Siddiqi claims that this dynamic system is Hamiltonian everywhere except on the skeleton. This implies that on skeletal points the field $\boldsymbol{F}$ is conservative, or $\nabla \cdot \boldsymbol{F}=0$. However, the total inward flux through the whole shape is non zero. In fact, the flux is proportional to the length of the boundary.

The divergence theorem states that the integral of the divergence of a vectorfield over an area is equal to the flux of the vector field over the enclosing boundary of that area. In our case,

$$
\int_{A} \nabla \cdot \boldsymbol{F} d \sigma=\int_{L} \boldsymbol{F} \cdot \boldsymbol{n} d l=\Phi_{A}(\boldsymbol{F}),
$$

where $A$ is any area, $\boldsymbol{F}$ is a field defined in $A, d \sigma$ is the area differential in $A, d l$ is the length differential on the border $L$ of $A$, and $\Phi_{A}(\boldsymbol{F})$ is the outward flux of $F$ through the border $L$ of the area $A$.

By virtue of the divergence theorem we have that, within the interior, there are points where the system is not conservative. The non-conservative points are those where the boundary trajectory is not well defined, i.e. where there are singularities in the evolution of the boundary. These points are the so-called shocks or skeleton of the shape- boundary. Shocks are thus characterized by locations where $\nabla \cdot \boldsymbol{F}<0$.

\subsection{Curvature in the Boundary Front}

Unfortunately, the hypothesis that the field $\boldsymbol{F}$ is conservative does not hold in general.

Let us consider an instant $t$ in the inward boundary evolution. The initial border has evolved under the eikonal equation to boundary front $S^{t}$ orthogonal 
in every point to $\boldsymbol{F}$. Pick a point $p \in S^{t}$, what is the value of $\nabla \cdot \boldsymbol{F}(p)$ ? Since the divergence operator is invariant under rotations, we can write $\nabla \cdot \boldsymbol{F}=\frac{\partial}{\partial v_{\|}} F+$ $\frac{\partial}{\partial v_{\perp}} F$ where $v_{\|}=\boldsymbol{F}(p)$ and $v_{\perp}$ is a normal vector orthogonal to $v_{\|}$. Since $\|F\|=1$ everywhere, $\frac{\partial}{\partial v_{\|}} F=0$. On the other hand $\frac{\partial}{\partial v_{\perp}} F(p)=-\kappa(p)$ where $\kappa(p)$ is the curvature in $p$ of the border front $S^{t}$ oriented so that $\kappa(p)$ is positive if the osculating circle is in the interior of the front. Hence, we have that $\nabla \cdot \boldsymbol{F}(p)=$ $-\kappa(p)$, that is, the divergence $\nabla \cdot \boldsymbol{F}$ is not always 0 as predicted by the HamiltonJacobi approach, but rather it is equal to the curvature of the front of the inward evolving boundary.

This result can be easily understood with an analogy from physics: Let us assume that a fluid flows from the border of the shape, which acts as a source, to the skeleton, which acts as a sink. If the fluid is incompressible, the fluid density never changes and the velocity field $\boldsymbol{F}$ is conservative everywhere except at point in the border and skeleton. On the other hand if the fluid is compressible, as soon as a curved front compresses the fluid, the density changes and the velocity field is not conservative anymore.

Let us pick a segment $d l(t)$ of the border front $S^{t}$. Let us assume this segment has average linear density $\bar{\rho}(t)$ (see Figure 1). Under the eikonal equation, at time $t+\Delta t d l(t)$ has evolved to $d l(t+\Delta t)$. Since all the points in $d l(t)$ are now

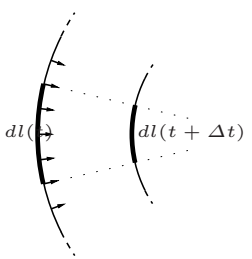
in $d l(t+\Delta t)$, the total mass of the two segments is the same, but if $d l(t)$ is curved the lengths of the segments are different: $\|l(t+\Delta t)\| \neq\|l(t)\|$. From this we obtain that the average density in $l(t+\Delta t)$ is $\bar{\rho}(t+\Delta t) \neq \bar{\rho}(t)$. Hence when the front is curved, the density is not constant and we have to take into account mass effects. That is: we have to resort to a more general conservation principle: the conservation of momentum.

\section{Momentum field}

Using this physical intuition we state that there is indeed a conservative field associated with the dynamics of the boundary: the momentum $\boldsymbol{M}=\rho \boldsymbol{F}$, where $\rho$ is the scalar field that assigns to each point the linear density of the boundary front, and hence $\nabla \cdot(\rho \boldsymbol{F})=0$,. After some algebra, we obtain the partial differential equation (PDE) $\nabla \rho \cdot \boldsymbol{F}=-\rho \nabla \cdot \boldsymbol{F}$. which goversn the density evolution in the flow field. Setting $\sigma=\log (\rho)$, we can write the above PDE as a function of the log-density $\sigma$ and hence $\rho \nabla \sigma \cdot \boldsymbol{F}=-\rho \nabla \cdot \boldsymbol{F}$.. Eliminating $\rho$ on both sides, we obtain:

$$
\nabla \sigma \cdot \boldsymbol{F}=-\nabla \cdot \boldsymbol{F}
$$

This is a transport equation which can be reduced to a set of ordinary differential equations (ODE) along the paths of the boundary points.

$$
\left\{\begin{array}{l}
\frac{d}{d t} \sigma(s(t))=-\nabla \cdot \boldsymbol{F}(s(t)) \\
\frac{d}{d t} s(t)=\boldsymbol{F}(s(t))
\end{array}\right.
$$


We can derive this equation by analyzing the change of density of segment $d l$ in Figure 1. We know that $\bar{\rho}(t)\|d l(t)\|=m$. where $d l(t)$ is the evolution of the border segment $d l$ at time $t, m$ is its mass, and $\bar{\rho}(t)$ and $\kappa(t)$ are the segment's average linear density and curvature at time $t$.

After a small interval of time $\Delta t$, the segment length will be $\|d l(t+\Delta t)\|=$ $\|d l(t)\| \frac{\kappa(t)}{\kappa(t+\Delta t)}+O\left(\Delta t^{2}\right)$, and the curvature $\kappa(t+\Delta t)=\frac{\kappa(t)}{1-\kappa(t) \Delta t}+O\left(\Delta t^{2}\right)$. From these equations and the conservation of mass, we have: $\bar{\rho}(t+\Delta t)-\bar{\rho}(t)=$ $\bar{\rho}(t) \frac{\kappa(t) \Delta t}{1-\kappa(t) \Delta t}+O\left(\Delta t^{2}\right)$. Taking the limit for $\Delta t \rightarrow 0$ and $\|d l\| \rightarrow 0$, we have:

$$
\frac{\frac{d}{d t} \rho(s(t))}{\rho(s(t))}=\kappa(s(t))
$$

where $s(t)$ is the trajectory under the eikonal equation of the limit point the segment $d l$ tends to as $\|d l\| \rightarrow 0$. Integrating (4) and using the fact that $\kappa(p)=$ $-\nabla \cdot \boldsymbol{F}$, we find: $\log (\rho(s(t)))=-\int_{0}^{t} \nabla \cdot \boldsymbol{F}(s(\tau)) d \tau$.

\section{Computing the density}

To obtain the momentum field we need to integrate the density field in the interior of the shape. Since images have a finite resolution, we need to discretize the solution in the image lattice.

One approach is to express the PDE (2) as a system of difference equations. The difference equations form a linear system that is then solved to obtain the $\log$-density $\sigma=\log (\rho)$. The problem with this approach is that the skeleton is a set of singularities of the momentum field, hence the density can have very different values at opposite sides of a skeletal branch. The result is that the linear system will have no solution and even trying to approximate a solution using a residual descent methods would force the density values the solution to oscillate wildly on points near the skeleton.

\subsection{Integration in Time}

In order to overcome this problem we need to be sure that the difference operator used in the equations never cross a skeletal branch. One way to guarantee this is to integrate the equation in the time domain: so that the formulae giving the value of $\rho$ at points in the boundary front at time $t$ reference values of $\rho$ only at points in the fronts at previous times. We can obtain this by integrating the ODE (3) along the paths of the boundary points.

We opt to use the second order Cranck-Nicolson method, that is, for each point $(x, y)$ in the interior of the shape, we have the equation:

$$
\sigma(s(t))=\sigma(s(t-1))-\frac{1}{2}[\nabla \cdot \boldsymbol{F}(s(t))+\nabla \cdot \boldsymbol{F}(s(t-1))] .
$$

Using this equation we can calculate the log-density at of a point of the border at time $t$ referencing only values of the log-density at points that belong the front at previous times. Since the evolution never crosses the skeleton, we are guaranteed not to cross skeletal branch through our calculations. 


\subsection{Integration in Space}

Equation (5) allows us to integrate the log-density $\sigma$ in the time domain along the path $s$, but we haven't shown how to calculate the path. Fortunately, we do not need to calculate every possible path. Let us assume that at time $t$ the boundary front passes through point $s(t)=(x, y)^{T}$, we can obtain a first order approximation of the position of that point at time $t-1$ as: $s(t-1)=\left(x-F_{x}, y-F_{y}\right)^{T}$. Using this approximation, we can write equation (5) in the domain of space instead of time:

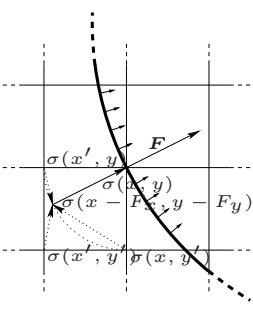

Fig. 2. Integration along the boundary path.

$$
\begin{aligned}
\sigma(x, y)=\sigma\left(x-F_{x}(x, y), y-F_{y}(x, y)\right) \\
\left.\quad-\frac{1}{2}[\nabla \cdot \boldsymbol{F}(x, y))+\nabla \cdot \boldsymbol{F}\left(x-F_{x}(x, y), y-F_{y}(x, y)\right)\right] .
\end{aligned}
$$

As shown in Figure 2, the point $(x, y)^{T}-\boldsymbol{F}(x, y)$ does not belong to the image lattice, hence we need to interpolate it using the values at the four corners of the square containing the point. We opt to compute the quantity $f(x+a, y+b)$ with $a, b \in[0,1)$ as: $\tilde{a} \tilde{b} f(x, y)+\tilde{b} a f(x+1, y)+\tilde{a} b f(x, y+1)+a b f(x+1, y+1)$, where $\tilde{a}=1-a$ and $\tilde{b}=1-b$.

Using Equation (6) we can compute the value of the log-density $\sigma(x, y)$ using values of $\sigma$ at points spanned by the boundary points before $(x, y)^{T}$. Hence, all we need to calculate $\sigma$ is to iterate equation (6) through the interior points by front arrival time, starting from the points that the boundary front reaches first, to the ones that it reaches last. Since the boundary is moving with constant unitary time, the time it takes the border front to reach a point $(x, y)^{T}$ is equal to its distance to the boundary.

Once we have the density to hand, we need to calculate the divergence of the momentum in every point of the image lattice. We opt to discretize Equation (2) using a second order approximation. Setting $\Delta \sigma=\sigma(x, y)-\sigma\left(x-F_{x}, y-F_{y}\right)$, we have:

$$
\begin{aligned}
& \nabla \cdot(\rho \boldsymbol{F})(x, y)=\Delta \sigma \exp \left(\sigma(x, y)-\frac{1}{2} \Delta \sigma\right) \\
+ & \frac{1}{2}\left[\nabla \cdot \boldsymbol{F}\left(x-F_{x}, y-F_{y}\right) \exp \left(\sigma\left(x-F_{x}, y-F_{y}\right)\right)+\nabla \cdot \boldsymbol{F}(x, y) \exp (\sigma(x, y))\right] .
\end{aligned}
$$

\section{Skeletonization}

Once the divergence of the momentum field is to hand, we can extract the skeleton. The extraction is performed by thinning the shape by removing border points that have energy absorption below a certain threshold and whose removal would not cause the shape to be split into two disjoint parts. The remaining shape is further thinned to a 1-pixel wide skeleton, paying attention to keep 
the shape connected and not to shorten the skeleton by eliminating endpoints. Expressed in pseudo-code the thinning process of shape $S$ is as follows:

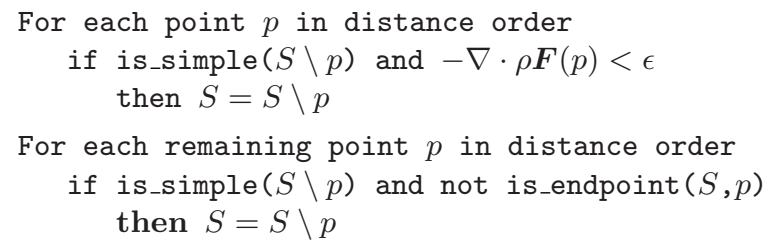

The predicate is_simple determines whether the shape is still connected after the removal of point $p$ by checking only the points in the neighborhood of $p$ : the shape $S \backslash p$ is connected if the point in the neighborhood of $p$, excluding $p$, are connected. Similarilly, is_endpoint determines whether $p$ is an endpoint only by inspecting the neighborhood of $p$ : the point is an endpoint if it has at most two neighboring points and those points are horizontally or vertically adjacent.

\section{Experimental Comparison}

In this section we try to characterize the differences between the HamiltonJacobi skeletonization method and our density-corrected approach. We start by providing a qualitative analysis of the difference in the divergence of the velocity and momentum fields. Secondly we provide an analysis of the noise and thresholding sensitivity of the two methods. Finally we provide a more quantitative analysis of the localization properties of the two skeletonization methods.

Figure 3 shows, for a few selected shapes in our database, the values of the divergence of the velocity field $\nabla \cdot \boldsymbol{F}$, $\log (\rho)$, and $\nabla \cdot \boldsymbol{\rho} F$. In this pictures white corresponds to a large positive values, black to a large negative value and 0 is represented by a $50 \%$ intensity gray.

It is very clear from the pictures that the divergence of the velocity field is not 0 in correspondence with a curved boundary. Furthermore, quantization in the localization of the border causes the initial border to be very jagged, and this highfrequency, low-amplitude noise is transported and amplified throughout the velocity field, yielding a noisy and poorly localized skeleton. Conversely, the density correction in the momentum field dampens the noise.

To counter quantization noise from the border, we need to smooth the distance

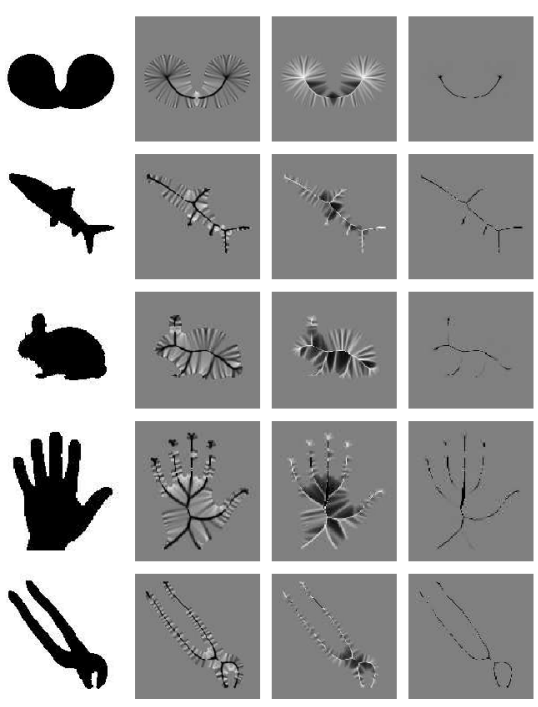

Fig. 3. Differences in the velocity and momentum fields. Left to right: shape, $\nabla \cdot \boldsymbol{F}, \log (\rho)$, and $\nabla \cdot \boldsymbol{\rho} F$ 
map and select an appropriate skeletonization threshold. Pick too big a smoothing radius or threshold and some branches of the skeleton will be thinned away, pick values too small and the extracted skeleton will have a lot of spurious branches (See Figure 4). In this section we characterize the effects on the extracted skeleton of the smoothing radius and the skeletonization threshold.

Figure 4 Displays the effects of very low (left) and very high (right) values of smoothing radius and skeletonization threshold on a test shape. The picture show, top to bottom, the divergence of the velocity field, the uncorrected HamiltonJacobi skeleton, the divergence of the momentum field, and the skeleton extracted using the densitycorrected method. what these picture show is that the density corrected method is much less sensitive to the amount of smoothing and the value of the threshold.

Next we characterize the localization properties of the skeleton extracted using the HamiltonJacobi and the new density corrected method on a
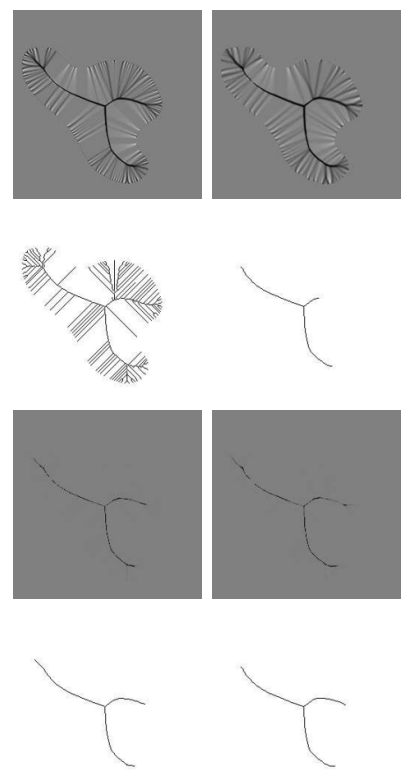

Fig. 4. The effect of smoothing on skeleton extraction. wide range of shapes. To this purpose we compute how the value of the divergence of the velocity and momentum field distribute over distance to the extracted skeleton. Figure 5 plots an histogram of how nonskeletal points distribute over distance and divergence value on our test shape. The figure shows that the Hamilton-Jacobi skeleton has a non-negligible amount of high divergence values even at high distance from the extracted skeleton.

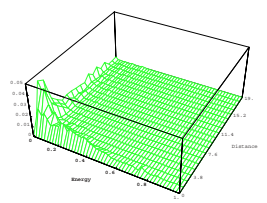

(a) Hamilton-Jacobi

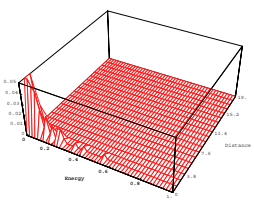

(b) Density corrected

Fig. 5. Histogram over value of (negative) divergence of the field and distance to skeleton

In an experiment we tried to quantify the localization of the skeleton on a database of shapes. To this purpose we used a database of 50 shapes and we histogrammed the distribution of field divergence over the distance to the skeleton for both the velocity and the momentum field. We then take, for each shape, the mean of this divergence-distribution as a measure of divergence-localization. 


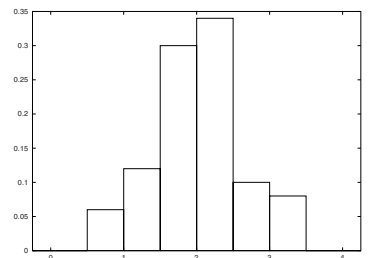

(a) Hamilton-Jacobi

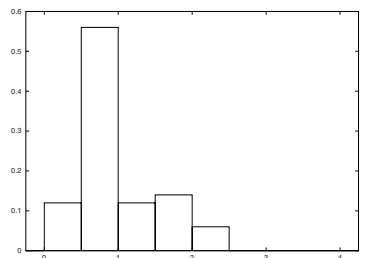

(b) Density corrected

Fig. 6. Histogram of divergence-localization of 50 shapes.

Figure 6 shows the histograms of the values of this divergence-localization measure over all the shapes in our database. On the left we see the localization histogram of the velocity field. The mean of this distribution is 2.52 , while the variance is 0.34 . The histogram on the right displays the localization properties of the momentum field. The mean of this distribution is 1.46 , while the variance is 0.28. The density correction clearly leads to a better localization of the skeleton.

\section{Conclusions}

In this paper we have given proof that a key hypothesis underpinning the Hamilton-Jacobi framework: the fact that the velocity field is conservative, does not hold in the presence of curved boundary. This is due to the increase of point density connected with a curved front of boundary evolution. In this paper we present an analysis which takes into account variations of density due to boundary curvature. This yields a skeletonization algorithm that is both better localized and less susceptible to boundary noise than the Hamilton-Jacobi method.

\section{References}

1. C. Arcelli and G. Sanniti di Baja. Ridge points in euclidean distance maps. Pattern Recognition Letters, 13:237-243, 1992.

2. H. Blum. Biological shape and visual science (part i). Journal of theoretical Biology, 38:205-287, 1973.

3. D. Macrini et al. View-Based 3-D Object Recognition using Shock Graphs. In $I C P R$, pages 24-28. IEEE Computer Society, 2002.

4. R. L. Ogniewicz and O. Kübler. Hierarchic voronoi skeletons. Pattern Recognition, 28(3):343-359, 1995.

5. S. J. Osher and J. A. Sethian. Fronts propagating with curvature dependent speed: Algorithms based on hamilton-jacobi formulations. J. of Computational Physics, 79:12-49, 1988.

6. M. Schmitt. Some examples of algorithms analysis in computational geometry by means of mathematical morphological techniques. In Geometry and Robotics, 1989. LNCS 391.

7. K. Siddiqi, S. Bouix, A. Tannenbaum, and S. W. Zucker. Hamilton-Jacobi skeletons. Int. J. of Computer Vision, 3:215-231, 2002.

8. K. Siddiqi and B. B. Kimia. A shock grammar for recognition. In $C V P R$, pages 507-513. IEEE Computer Society, June 1996.

9. H. Tek and B. B. Kimia. Symmetry maps of free-form curve segments via wave propagation. In ICCV, pages 362-369. IEEE Computer Society, September 1999. 